\title{
On utrocular discrimination
}

\author{
RANDOLPH BLAKE \\ Cresap Neuroscience Laboratory, Evanston, Illinois 60201
}

and

ROBERT H. CORMACK

New Mexico Institute of Mining and Technology, Socorro, New Mexico 87801

\begin{abstract}
Observers with good stereoacuity judged which eye received sine-wave grating patterns in a two-category forced-choice procedure. Large individual differences were found, but for most observers reliable discrimination was achieved at low spatial frequencies. No observer could perform the task above chance levels at high spatial frequencies. Discrimination was unaffected by retinal location, grating orientation, grating contrast, stimulus duration, or practice with feedback. Among observers who could perform the task, the following results were obtained: (1) Introduction of high spatial frequency components did not interfere with performance so long as a low spatial frequency component was present. (2) When gratings of low equal spatial frequency were presented to both eyes simultaneously at different contrast levels, observers could identify which eye received the higher contrast. (3) At low spatial frequencies, observers could distinguish monocular from binocular presentation. (4) Temporal frequency variations (counterphase flicker) influenced performance for some observers. Binocular summation and interocular transfer were unaffected by the spatial frequency variations which modulate utrocular discrimination. A new procedure for measuring stereopsis was developed which made possible comparison of utrocular discrimination with stereopsis at specific spatial frequencies. Stereopsis appeared mildly affected by spatial frequency.
\end{abstract}

When queried, most people claim that it is impossible to tell with which eye they are actually seeing when both eyes are open. Typically, the question is met with puzzlement, for common experience tells us that we are seeing with both eyes, simultaneously. Yet there are some individuals who report the knack of seeing with one eye alone without evidencing any noticeable change in the posture of the "blind eye." These individuals quite frequently have histories of disturbances in binocular vision, such as strabismus early in life, and typically perform poorly on tasks requiring stereoscopic depth perception, a condition referred to as stereoblind. By analogy with stereoblind animals (Blake \& Hirsch, 1975; Packwood \& Gordon, 1975), Lema and Blake (1977) speculated that such people might have predominantly monocular neurons in the visual cortex in place of the full complement of cortical binocular neurons which most of use presumably possess.

To test this speculation, we (Blake \& Cormack, 1979) measured utrocular discrimination ${ }^{1}$ in normal

This research was supported by grants from NSF (BNS 75-17073) and NIH (EYO1596). We are grateful to Robert Fox, Randall Overton, and Robert Sekuler for helpful discussion and to John Camisa and William Martens for their many hours of generous service as observers. Requests for reprints should be sent to Randolph Blake, Cresap Neuroscience Laboratory, Northwestern University, Evanston, Illinois 60201. and stereoblind human observers. Our results confirmed that stereoblind humans could reliably report which one of the two eyes had received visual stimulation under conditions which rendered the performance of normal observers unreliable. Somewhat to our surprise, however, we also found that under certain conditions many normal observers with excellent stereopsis could make accurate utrocular judgments, too, just as well as the stereoblind individuals. Intrigued by this latter finding, we proceeded to study utrocular performance of normal observers in further detail, the aim being to gain some further understanding about the workings of the binocular visual system. The results from these experiments are reported here.

This paper begins with a review of the meager literature on the problem of utrocular discrimination. Next, we expand on the neurophysiological speculations which have guided our thinking about the possible bases of utrocular discrimination. In Part 1 of the results, we examine the stimulus conditions which influence the ability of normal observers to judge accurately which of the two eyes has received stimulation. In Part 2, we attempt to relate our findings to performance on other psychophysical tasks involving binocular vision. We conclude by exploring the methodological and theoretical implications of our findings. 


\section{Background}

Interest in the problem of utrocular discrimination originally emerged within the context of theories of stereopsis. Prior to the discovery of disparity-selective binocular neurons, it was fashionable to speak of stereopsis in terms of the combination of two distinct cortical images, themselves the projections from the two eyes. Now, in order to distinguish crossed from uncrossed disparities, it would be necessary for the brain to preserve and somehow code eye-of-origin information; otherwise, stereopsis would be ambiguous within the scheme of these so-called projection theories (Gregory, 1978).

The discovery of binocular cortical neurons with different preferred disparities (Barlow, Blakemore, \& Pettigrew, 1967; Nikara, Bishop, \& Pettigrew, 1968) has changed the way we think about stereopsis. It no longer is necessary to deal with the eye-of-origin problem, for the sign of the disparity (crossed vs. uncrossed) is an inherent property of the receptive field layout of binocular neurons. Unlike the earlier image projection theories, models of stereopsis based on disparity detectors do not require that eye-of-origin information be retained within the visual nervous system. As a consequence of this new way of thinking about binocular interaction, the problem of utrocular discrimination has been relegated to the sidelines and has received little theoretical or empirical attention. In this paper, we hope to demonstrate that utrocular discrimination still offers a fruitful means for learning about binocular neural interaction.

Turning now to prior studies of utrocular discrimination, we are aware of only a few studies (Barrett \& Williams, 1965; Enoch, Goldmann, \& Sunga, 1969; Pickersgill, 1961; Smith, 1945; Thelin \& Altman, 1929) that have dealt with utrocular discrimination in this century. All these studies employed the same basic approach, whereby a stimulus is presented to just one eye without informing the observer which eye will actually receive it. The observer is required to report which of the two eyes "saw" the stimulus, and above-chance performance over a series of trials is taken as evidence for utrocular discrimination. ${ }^{2}$ With one exception (Barrett \& Williamson, 1965), the stimuli in these experiments have consisted of small spots of light which appeared against a dim, sometimes dark, background; the study by Barrett and Williamson (1965) employed real, three-dimensional objects. Of the five studies, only the one by Enoch et al. (1969) was designed to eliminate or control many of those extraneous cues which could enable a clever observer to distinguish which eye was stimulated. Such potentially confounding cues can arise from astigmatism, chromatic aberration, fixation disparity, or anisotropies in acuity between the two eyes, not to mention subtle differences between the physical stimuli presented to the left eye vs. right eye.
The following summary of findings therefore relies heavily on the work of Enoch et al. and applies only to the case of briefly flashed spots of light.

There seems to be the consensus that some, but not all, normal observers can report which of the two eyes has received a light flash with greater than chance accuracy. Many of these observers describe the mediating cue as a feeling of "strain" or "interference" in the stimulated eye. In view of these descriptions, it is interesting that paralysis of pupillary reflex and accommodation has no effect on utrocular discrimination. Practice on utrocular discrimination tasks can produce some improvement in performance, but it is clear that practice alone will not elevate all observers to above-chance levels. Among observers who succeed at the task, performance appears to improve somewhat as exposure duration increases to about 300-500 msec, and levels off thereafter; this effect of duration is not dramatic, however. In general, these experiments provide little information concerning experimental manipulations which strongly influence utrocular discrimination, nor have they identified variables which show a clear relationship to the ability. Moreover, it is not at all obvious why there exists such large individual differences in the ability to make eye-of-origin judgments. We suspect that this confusing state of affairs has made its own contribution to the paucity of attention to the problem of utrocular discrimination.

\section{Neural Considerations}

It will be useful at this point to outline what would seem to be the minimal neural requirements for successful utrocular discrimination. We start with the following assumption: In order for stimulation of the left eye to be psychophysically discriminable from stimulation of the right, the neural events produced by left-eye stimulation must be distinguishable from those accompanying right-eye stimulation. This, of course, represents a specific instance of Brindley's (1970) general psychophysical linking hypothesis. Now there can be no doubt that the initial neural events associated with left-eye stimulation are different from those associated with stimulation of the right, for we are dealing with different receptor surfaces and optic nerves; prior to visual cortex, neural information from the two eyes largely remains segregated, which means that a place code of sorts could potentially support utrocular discrimination. However, we typically think of visual detection and discrimination as involving processes beyond just these first few synapses of the visual nervous system, so it becomes necessary to think in terms of neural events within cortical areas or in midbrain structures. At these higher visual stages, it is very likely that wholesale neural interaction between the two eyes has served to obliterate the potential place code available earlier 
in the visual pathways. Microelectrode recordings have shown that many neurons in visual cortex and superior colliculus receive input from both eyes, which means that eye-of-origin information is no longer available within a single binocular neuron. It is also the case, however, that binocular neurons vary in terms of their ocular dominance, with some cells much more responsive to stimulation through one eye than through the other. It is conceivable that eyeof-origin information could be contained in a complex way within the pattern, or distribution, of activity among a population of neurons with varying degrees of ocular dominance.

A simpler mechanism might involve the extreme case of ocular dominance wherein neurons are activated through one eye only. An ocular dominance profile which emphasizes these extremes of the distribution should serve to enhance the probability of successful utrocular discrimination. It was this idea which prompted us to compare utrocular performance of normal and stereoblind humans. While our results (Blake \& Cormack, 1979) were consistent with this idea, we also discovered that the performance of normal observers exceeded chance levels under some circumstances. This intriguing result does not force us to abandon our initial working model based on the concept of ocular dominance, but it does indicate that the model requires some further elaboration. The experiments described in this paper were designed with this as the goal.

\section{METHODS}

\section{Visual Displays and Apparatus}

In all the experiments in this paper, the visual displays consisted of one-dimensional grating patterns generated electronically on two matched cathode-ray tube (CRT) monitors (Tektronix 604, P-31 phosphor). In most experiments, the gratings were sinusoidal in waveform, although square-wave luminance profiles and one dimensional random noise were employed in two of the experiments.

Stereoscope. The two CRT displays were presented separately to the two eyes via a mirror stereoscope, with a viewing distance of $114 \mathrm{~cm}$. At this distance, the rectangular borders of the CRT subtended to $7^{\circ} \times 5^{\circ}$. The observer viewed the CRTs through natural pupils (except where noted), with the head securely positioned on a dental impression board. Optical elements (prisms, lenses, filters, artificial pupils) could be mounted in a binocular eyepiece, through which the observer viewed the CRTs. A pair of adjustable front-surface mirrors could be rotated to bring the two CRT displays into proper binocular alignment.

Grating patterns. A raster was created on each CRT screen by applying a $100-\mathrm{kHz}$ triangle wave to the vertical plates of the CRT while maintaining a $100-\mathrm{Hz}$ frame rate. This produced uniform illumination of the CRT screen, with an average luminance of $7 \mathrm{~cd} / \mathrm{m}^{2}$. The beam current (Z-axis) of each CRT could be modulated by the sine-wave or the square-wave output of either of two identical function generators (Clark Hess Model 748) both function generators were time-locked to the sweep signal applied to the horizontal plates of the CRT. Before application to the Z-axis of the CRTs, each modulating signal was passed through a pair of decade attenuators (Hewlett Packard, Model
350D) and then fed into an electronic shaping circuit which served to gate the modulating signals on and off gradually according to a Gaussian function with a space constant of $1.5^{\circ}$ of visual angle. This shaping circuit was time-locked to the $100-\mathrm{Hz}$ sweep signal, such that the modulating signal rose to its full amplitude and then fell to the dc level with every sweep. With this arrangement, the grating pattern produced on the CRT was amplitude modulated over space in a manner which vignetted the grating at the lateral borders of the display; this served to minimize the harmonic distortions which naturally arise when a repetitive grating pattern is abruptly truncated by a border (Kelly, 1970). In addition, by confining the pattern just to the central portion of the CRTs, potential extraneous cues due to fixation disparity were minimized.

The spatial frequency and the contrast of the gratings could be varied independently without affecting the overall luminance. The gratings could be flickered in counterphase by amplitude modulation (AM) of the output of the function generators with a low-frequency sinusoid. With this form of flicker which we employed in the experiments dealing with temporal frequency, the light and dark bars of the grating interchanged positions continuously. In the absence of the low-frequency AM signal, the bars of the grating appeared stationary on the screen. A linear summing amplifier could be used to mix different sinusoids in order to generate a complex grating composed of several different spatial frequencies. In all except one experiment, the bars of the gratings were vertically oriented; for that experiment, horizontal gratings were produced by interchanging the inputs to the vertical and horizontal deflection plates of the CRTs.

Dynamic visual noise. In one experiment, a complex display consisting of one-dimensional random noise was employed in place of the usual simple grating. This random-noise display was created by using the output from a noise generator (GrasonStadler Model $1285 ; 20-200-\mathrm{kHz}$ bandwidth with less than $.5 \%$ total harmonic distortion) to modulate the Z-axis beam intensity. This display consists of a dynamic array of many gratings which change in spatial frequency and phase continuously over time. The average contrast of the display at any one moment can be estimated by taking the RMS voltage necessary to produce a simple sinusoidal grating of arbitrary contrast $\mathrm{m}$, and dividing this voltage by $1 / 2$; the resulting voltage will yield random noise, the average contrast of which will be $m$ or less $95 \%$ of the time (Stromeyer \& Julesz, 1972).

Timing circuit. For all the experiments in Part 1 and some of those in Part 2, the gratings were briefly exposed for durations that are given in the results. The display was presented by triggering an electronic timer which was preset for the desired duration. Rather than being turned on and off abruptly, the gratings were gradually introduced and withdrawn by the use of a shaped rise/fall gate with programmable time constants. The timer which actuated this gate and controlled the duration of exposure could be triggered by depressing a button which was accessible to the observer.

\section{General Procedure}

This section gives procedural information common to various groups of experiments.

Utrocular discrimination task. In these experiments, percentcorrect performance was recorded in a two-alternative (right-eye/ left-eye) forced-choice procedure. The observer was required to report which one of the two eyes saw a grating pattern. The observer initiated a trial by depressing a pushbutton which immediately triggered the brief presentation of a pattern on one of the CRTs. The observer then indicated which of the eyes received the display by operating a two-position lever switch; when uncertain, the observer was required to guess. Trial-by-trial feedback was not given (except where noted). In all experiments, the observer was informed of the stimulus condition (i.e., spatial frequency, flicker rate, orientation) under test and executed a number 
of practice trials to become familiar with the specific stimulus. The contrast of the test pattern always was well above threshold visibility. Except where noted, observers fixated the center of the display. Trials were administered in blocks of 50 or 100 , and within a block the eye receiving the pattern was randomly ordered from trial to trial. The order of conditions was counterbalanced across blocks. The sequencing of trials and collection and analysis of data were performed by a PDP-8I computer.

Throughout the course of the experiments, several measures were taken to promote good fusion and to insure equality of the displays to the two eyes. The sharp borders of the CRTs provided potent fusion stimuli. The cover/uncover test was employed to achieve accurate binocular alignment of the CRT screens, thereby minimizing fixation disparity. In order to place the gratings in precisely the same positions within the CRT screens, we adjusted the relative phase of the two gratings until the observer reported that the fused pattern appeared exactly in the plane of the CRT face. In order to match the apparent contrasts of the two gratings, we used an interocular flicker method in which the grating was alternated rapidly from one CRT to the other. Grating contrast was adjusted until the observer reported minimal flicker. Reduction screens in the optical paths eliminated extraneous visual stimuli.

Contrast adjustment method. Two experiments in Part 2 involved measuring contrast thresholds under conditions which allowed the assessment of binocular summation and interocular transfer. In both instances, there was an initial 2-min adaptation period to either an uncontoured display (binocular summation) or a high-contrast grating pattern (interocular transfer). Following the adaptation period, the observer adjusted the contrast of a test grating by turning a precision potentiometer until the contours of the display were just barely visible. During this test period, the test grating and the adaptation display were repetitively interchanged for 3 and $5 \mathrm{sec}$, respectively. The test and adaptation displays were introduced and withdrawn gradually (time constant of $250 \mathrm{msec}$ ) and appeared stationary $(0 \mathrm{~Hz})$. Once a satisfactory setting was achieved, the observer triggered a printout of the contrast value. Six threshold settings were made for each stimulus condition and the arithmetic mean was taken as the visibility threshold. Observers were encouraged to perform the adjustments carefully and to maintain the same criterion throughout. The observers tested on this task were very consistent in their performances; the standard error of the mean typically was no more than $.015 \log$ unit.

Stereopsis test. A nulling procedure, whereby an observer adjusted a stereoscopic grating to lie in the frontoparallel plane, was employed to assess stereoacuity. With this technique, the two eyes viewed a pair of vertical sinusoidal gratings, one of fixed spatial frequency and the other of variable spatial frequency; both gratings were of $10 \%$ contrast. By turning a precision potentiometer, the observer varied the dc voltage applied to the FM input of the right channel function generator, thereby changing the spatial frequency of the right-eye grating. The observer was instructed to adjust the display to eliminate the stereoscopic tilt sensation which occurs when the two eyes receive slightly different spatial frequencies (Blakemore, 1970; Fiorentini \& Maffei, 1971). The observer could take as long as necessary to perform each setting, and pressed a button to print out the final value. Practice trials were given before each series of 10 trials was made.

\section{Observers}

A total of 10 observers participated in one or more of the experiments reported here. All of these individuals $(3$ females and 9 males ranging in age from 19 to 44 years) have normal or correctedto-normal visual acuity and stereopsis, as assessed by the modified Orthorater. Observers with optical corrections used them during the experiments.

\section{RESULTS}

\section{Part 1}

In our initial work on utrocular discrimination (Blake \& Cormack, 1979), we found that some individuals with normal stereopsis were able to judge correctly which eye received stimulation under certain stimulus conditions, namely at lower spatial frequencies. The series of experiments in Part 1 was designed to examine this observation in more detail and to discover, if possible, those variables which contribute to differential performance at low vs. high spatial frequency. Accordingly, all the data reported in this part were obtained using the two-alternative forced-choice procedure and are expressed as percent-correct values.

Spatial frequency. Our first experiment measured utrocular performance over a 3-octave range of spatial frequencies. Grating contrast was $10 \%$, and the exposure duration was $350 \mathrm{msec}$; the grating always was vertically oriented. Results from this experiment are shown in Figure 1, which plots percent correct as the function of spatial frequency. At the lowest spatial frequency, all observers except R.C. scored above chance, while at the highest spatial frequen-

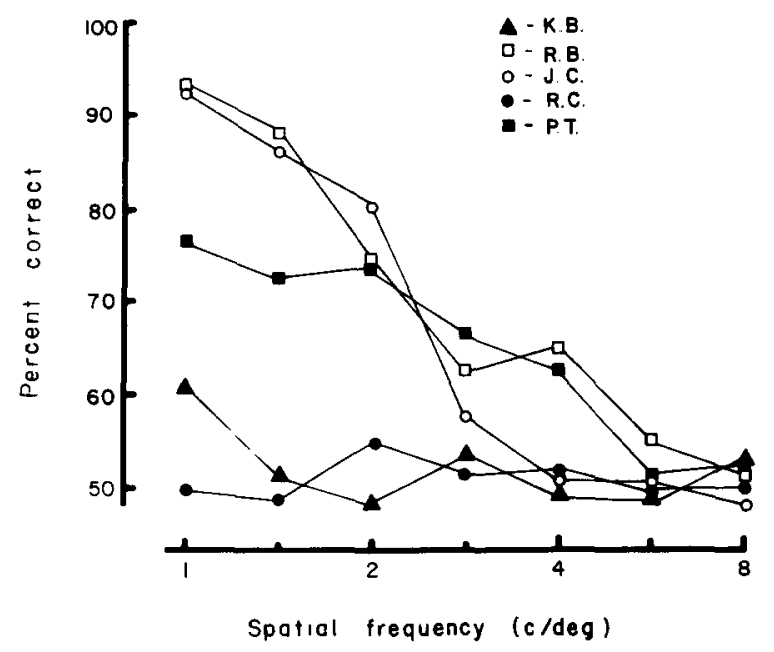

Figure 1. Utrocular discrimination as a function of grating spatial frequency. The various symbols represent different observers. In this and most of the following figures, utrocular performance is expressed in terms of percent correct. As this was a twoalternative forced-choice task, $50 \%$ represents chance performance. Unless noted otherwise, each point summarizes the results from at least 100 trials. In none of the figures have we plotted confidence intervals around the percent-correct values. These intervals are, however, straightforward to compute using the binomial distribution or to approximate using the normal distribution (since $n \geqslant 100$ in these figures). For purposes of reference, we have derived $95 \%$ confidence intervals for several representative performance levels, each based on $\mathrm{n}=100$. For chance performance, $50 \%$, this interval is \pm .098 ; for $75 \%$ correct, the interval is \pm .084 ; for $90 \%$ correct, the interval is \pm .058 . 


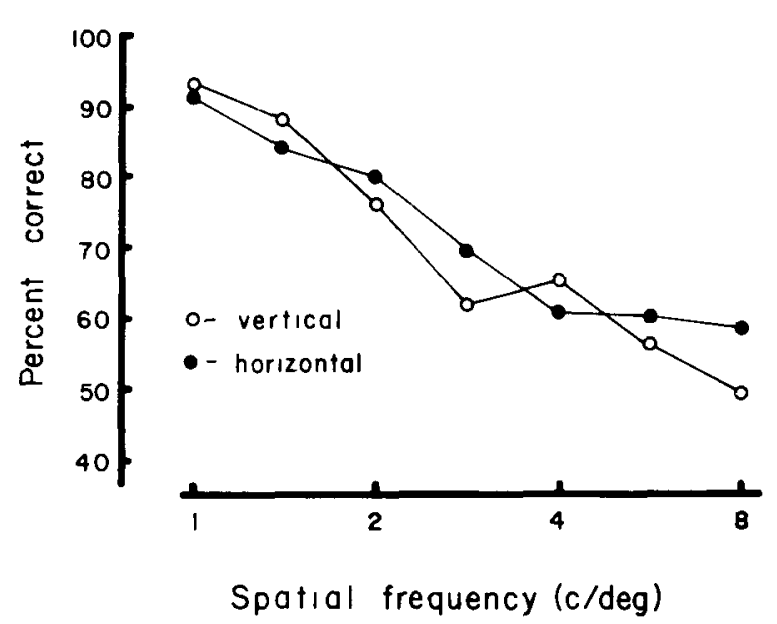

Figure 2. Utrocular discrimination for vertical and horizontal gratings at various spatial frequencies. The observer was R.B.

cies, the performance of all observers was reduced to the level of guessing. We also have tested R.B. and R.C. at even lower spatial frequencies, .71 and $.50 \mathrm{cycles} / \mathrm{deg}$. At these values, performance remained high for R.B. and continued at chance levels for R.C. Incidentally, the same pattern of results was found when the test grating was oriented horizontally. Figure 2 shows the performance of observer R.B. for horizontal (filled circles) and for vertical (open circles replotted from Figure 1). Similar measurements for horizontal were obtained for observer R.C., and he continued to perform at chance levels at all spatial frequencies. Thus, it seems clear that the relationship between spatial frequency and utrocular discrimination is identical for horizontal and vertical contours. In the remaining experiments, we employed vertical gratings exclusively.

We had occasion to test five other observers with normal stereopsis at 1 and 8 cycles/deg. All showed chance performance at $8 \mathrm{cycles} / \mathrm{deg}$, and four of the five showed significant improvement at $1 \mathrm{cycle} / \mathrm{deg}$.

These results confirm our initial observation that, among those people capable of utrocular discrimination, performance depends on spatial frequency. This outcome, in turn, raises a couple of interesting possibilities concerning the basis of this effect of spatial frequency. For one thing, it could be that high spatial frequencies somehow operate as noise which serves to mask eye-of-origin information, perhaps in a manner analogous to the high-frequency masking effects described by Harmon and Julesz (1973) in the case of pattern recognition. According to this notion, then, a test stimulus containing both low and high spatial frequencies should yield poorer utrocular performance than a stimulus composed only of a low spatial frequency. We tested this possibility by comparing percent correct for a 1-cycle/deg grating of sinusoidal waveform with that measured using two other, more complex displays. One display was a 1 -cycle/deg square-wave grating which contains energy at all the odd higher harmonics $(3,5,7 \ldots$ cycles/ deg) as well as at the fundamental spatial frequency, $1 \mathrm{cycle} / \mathrm{deg}$. The other display consisted of onedimensional noise, a stimulus which contains a broad spectrum of spatial frequencies ranging from low to high. (With the square-wave pattern, the amplitude of the higher harmonics is lower relative to the fundamental, but in the case of the noise display, all the frequency components are of the same average amplitude.)

As shown in Figure 3, these three different visual displays ( 1 cycle/deg sine, 1 cycle/deg square, noise) yield comparable performance on the utrocular discrimination task. Evidently the presence of high spatial frequencies neither aids nor interferes with the ability to discriminate which of the two eyes has received stimulation. Rather, it appears that low spatial frequencies represent the source of information necessary for this judgment.

The results in Figure 3 also indicate that it is the harmonic content of the pattern which is really the crucial stimulus variable mediating successful utrocular discrimination, and not the apparent period, or size, of the contours of a low spatial frequency grating. This conclusion follows from a comparison of the performance with the 1 -cycle/deg square-wave pattern and that with a complex grating which resembles the square-wave pattern in terms of its periodicity but which is composed of higher spatial frequency components only. This complex grating, known as a

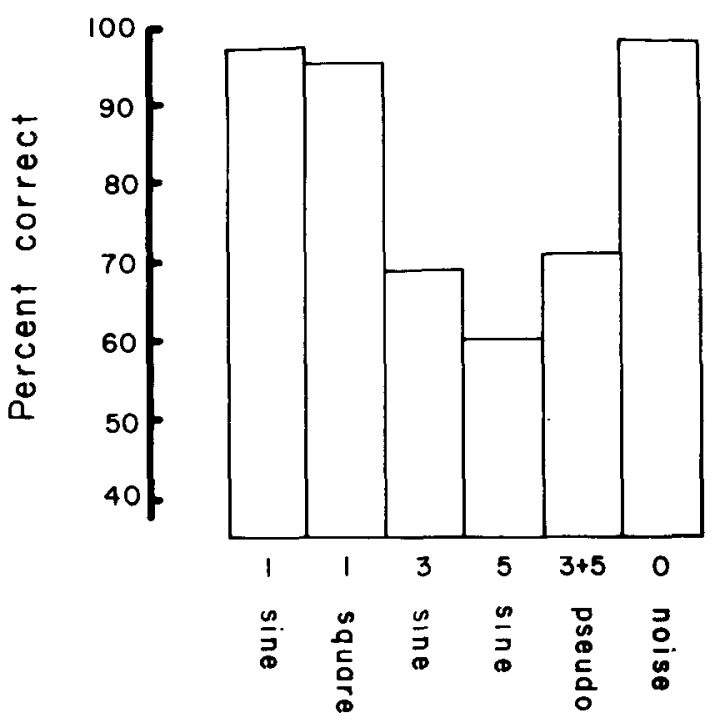

Fundamentals and waveform

Figure 3. Utrocular discrimination for six different waveforms. Numbers below the histograms give the spatial frequency of the waveform in cycles/degree (R.B.). 
pseudo-square-wave (Campbell, Howell, \& Robson, 1971), was generated by adding electronically gratings of 3 and 5 cycles/deg. By appropriately arranging the relative phase and amplitude of the two patterns, it was possible to produce a complex grating which appeared to contain coarse bars similar in size to those of the genuine square wave. But, as can be seen in Figure 3, utrocular discrimination with the pseudosquare-wave was inferior compared to that with the true square wave, despite their resemblance phenomenally, and instead was equivalent to performance measured for either the 3- or 5-cycle/deg sine component alone. These results indicate that successful utrocular discrimination depends upon the presence of energy at low spatial frequencies; large contours of phenomenal origin are alone insufficient to support consistently accurate performance. Incidentally, it is of some interest that simultaneous presentation of 3 and 5 cycles/deg fails to improve performance relative to the situation where only one of these gratings is presented. The absence of even probability summation indicates that the two components of the complex grating do not act as independent inputs, at least in terms of their contributions to utrocular discrimination.

Energic variables. The results presented so far were obtained using grating patterns which were well above threshold visibility, by almost 2 log units. To determine whether utrocular discrimination would deteriorate as the test pattern approached threshold, we measured performance as the function of grating contrast and duration of presentation.

Figure 4a gives the outcome of testing over almost a 1-log-unit range of contrast values, with contrast expressed in terms of decibels above threshold ( $20 \mathrm{~dB}$ equals $1.0 \mathrm{log}$ unit). The contrast threshold was determined using an adjustment technique (see Methods). The open symbols give the results for 1 cycle/deg, and the filled symbols represent the outcome for $8 \mathrm{cycles} / \mathrm{deg}$. Clearly, variations in grating contrast fail to influence performance at either spatial frequency; discrimination remains excellent at the low spatial frequency, even when the grating is barely visible, and remains uniformly poor at the high spa-

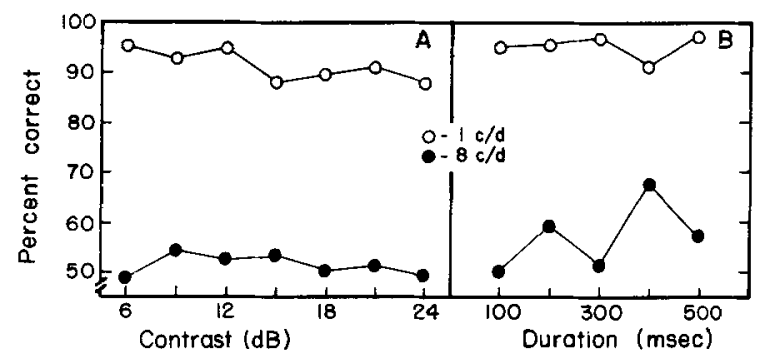

Figure 4. Utrocular discrimination as the function of grating contrast (A) and exposure duration (B) (R.B.).

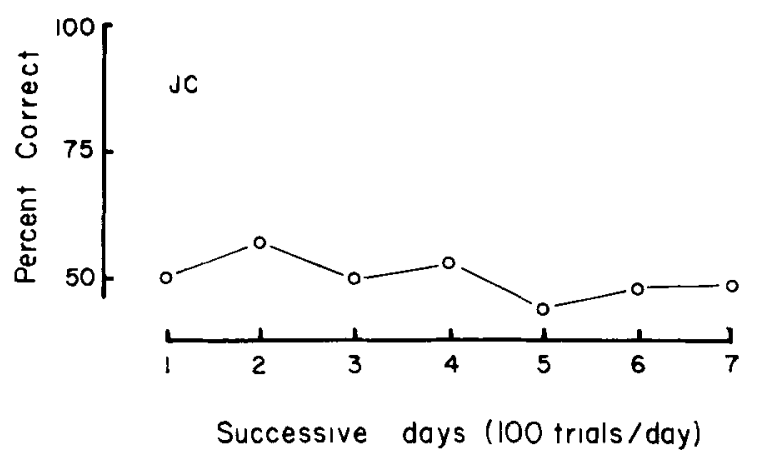

Figure 5. Utrocular discrimination at 6 cycles/deg as a function of amount of practice with feedback (J.C.).

tial frequency. This same pattern of results was obtained for observers J.C. and P.T.

Variations in the duration of presentation are similarly ineffective in terms of modulating utrocular performance. Using the same set of symbols, Figure $4 \mathrm{~b}$ plots performance at low and high spatial frequencies as a function of grating duration. For this experiment, the shaped rise/fall gate controlling the onset and offset of the grating was set at $100 \mu \mathrm{sec}$, the minimum value which could be achieved with this device. Over the range of durations studied, performance was virtually constant for observer R.B. Sampling fewer values but over the same range of durations, we found comparable results for observers W.M. and P.T. Observer R.C., who performed at chance levels at all spatial frequencies in our first experiment, showed no improvement in this experiment; his performance at 1 and 8 cycles/deg remained near $50 \%$ over the complete sample of durations.

Practice. Previous experiments (Enoch et al., 1969; Thelin \& Altman, 1929) have found a modest improvement in utrocular discrimination with practice. Naturally, we wondered whether the poor performance at high spatial frerquencies in our paradigm could be remedied by training. To examine this possibility, we tested observer J.C. daily for 1 week, with at least 100 trials/day. The test grating was 6 cycles/ deg and .10 in contrast. After his response on each trial, he was informed of the correctness of his response. The results from this prolonged series of trials are shown in Figure 5. They provide no evidence of improvement over the 1-week period.

Additional evidence against a practice effect is provided by the stability in performance of several observers throughout the course of our studies. In particular, observers R.B., R.C., J.C., and P.T. each completed several thousand trials. Although these trials were without immediate feedback, they did furnish the observer thorough familiarity with the displays and they often repeated identical conditions. Despite this extensive experience with the task, there was no real trend toward improvement at the higher 
spatial frequencies. This indicates that poor performance on this task stems from the absence of utrocular information, and it strengthens our conviction that extraneous cues are not contributing to successful performance at low spatial frequencies.

Ocular reflexes. Observers who successfully make utrocular discriminations often report that their judgments are based on a sensation which seems to be localized in the stimulated eye itself. This sensation is variously described in terms of a strain, a squeezing effect, a feeling of touch in the eye, etc. One observer described the feeling as somewhat analogous to the sensations which accompany sudden exposure to a mild source of glare. Of course, with our displays there is no change in overall luminance upon presentation of the test grating, so we assume that there is no change in pupil size, which, in any event, should be consensual. Still, in view of these phenomenal descriptions, it seemed worth examining the possible contribution of reflexes of the intrinsic ocular muscles.

To accomplish this, we measured utrocular discrimination at 1 and 6 cycles/deg before and after application of a briefly acting cycloplegic $(1 \%$ cyclopentolate hydrochloride) to both eyes of observer J.C. For these measurements, the observer viewed the display through 3-mm artificial pupils; spectacle lenses were used to correct for the paralysis of accommodation. Testing following application of the cycloplegic was not initiated until all signs of pupillary motility and accommodative response were absent.

The results from this experiment are shown in Figure 6, which plots percent correct for three successive blocks of 100 trials prior to (open symbols) and following (filled symbols) paralysis of the pupil and accommodation. Clearly, this manipulation had no

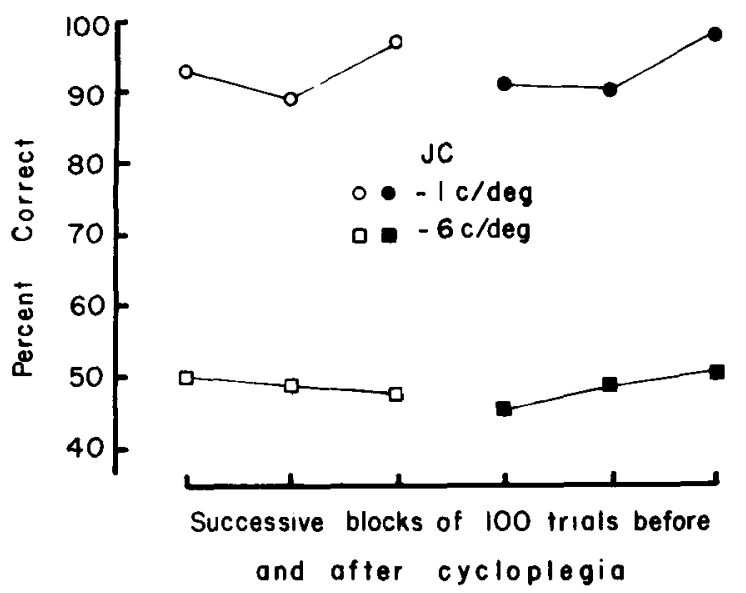

Figure 6. Effect of paralysis of intrinsic eye muscles on utrocular discrimination for two spatial frequencies. Open symbols plot performance before paralysis (J.C.). effect on the observer's ability to make utrocular judgments at the low spatial frequency. Nor, according to J.C., did it serve to alter the definite sensation which was associated with stimulation at that frequency. We can, therefore, rule out potential cues associated with changes in accommodation or pupil size. Of course, cycloplegia simply prevents these reflexes from being executed but does not eliminate the command signals responsible for their occurrence (i.e., efferents from oculomotor nuclei). It is conceivable that such command signals in some way provide eye-of-origin information. It is not obvious, however, why such information should be available only in response to low spatial frequencies. ${ }^{3}$

Retinal eccentricity. Having thoroughly convinced ourselves that the difference in utrocular performance at low and high spatial frequenices reflects some property of the visual system and not simply some artifact of the task or the display, we set out to examine a couple of variables which could shed additional light on the neural basis of this difference.

The following experiment measured utrocular discrimination for 1 and 8 cycles/deg using eccentric fixation. It is known that the fovea is more sensitive to high spatial frequencies than are more peripheral portions of the retina (e.g., Berkley, Kitterle, \& Watkins, 1975). In view of this, we wondered whether the effects of spatial frequency on utrocular discrimination would vary when the test grating was displaced away from the fovea such that it fell onto more peripheral regions of the retina. To test this possibility, we measured utrocular performance while the observer fixated the midpoint of the lateral border of the CRT display. This had the effect of confining the test display to one hemiretina (nasal or temporal, depending on which eye received the pattern). Because of the spatial vignetting, the grating itself did not extend to the borders of the CRT, which meant that the nearest visible portion of the pattern fell approximately $1.5^{\circ}$ into the periphery.

The results are plotted in Figure 7 in the form of bar histograms showing percent correct for three fixation conditions; for the condition labeled "right," the observer stared at the left-hand edge of the CRT, and vice-versa for "left." For this observer, variations in fixation had no influence on the difference in performance at low and high spatial frequencies. For observer R.C., who was tested in a similar fashion, eccentric fixation failed to improve his uniformly poor performance at these two spatial frequencies. We conclude, therefore, that the neural machinery subserving successful eye-of-origin judgments is not differentially distributed to nonfoveal regions of the retina, at least for that portion of the periphery examined.

Temporal frequency. Two considerations led us to study the effects of temporal modulation (i.e., flicker) 


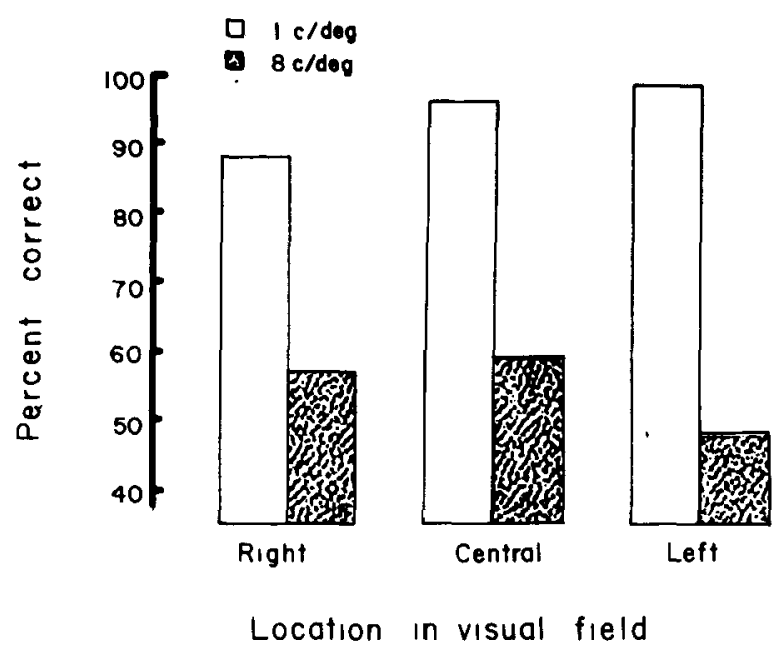

Figure 7. Utrocular discrimination as a function of retinal location of the grating pattern. The "right" condition refers to the case where the grating appeared to the right of fixation (R.B.).

on utrocular discrimination. For one thing, earlier papers which reported successful eye-of-origin performance used briefly flashed targets with abrupt onset and offset. Now this stepwise modulation of energy over time means, of course, that each stimulus presentation was rich in high temporal frequency components. Except for our experiment on duration, we have introduced and withdrawn the test grating according to a Gaussian envelope, which contains no high-frequency components. It is conceivable that a stimulus containing higher temporal frequencies might improve utrocular discrimination. The second consideration arises from the recent evidence, both electrophysiological and psychophysical, for two general classes of visual neurons which can be distinguished on the basis of their spatial and temporal response properties. From physiological experiments, it is known that one class, the so-called Y-cells, responds primarily to lower spatial frequencies, while the other class, the X-cells, responds best to higher spatial frequencies. In terms of their temporal properties, the $\mathrm{Y}$-cells, unlike $\mathrm{X}$-cells, respond vigorously to higher rates of temporal modulation. (See Ikeda \& Wright, 1974, for a review of these cell types.) There is psychophysical evidence suggesting that these two relatively distinct classes of neurons can be selectively activated by appropriate selection of spatial and temporal frequency (e.g., Kulikowski \& Tolhurst, 1973). For instance, a stationary grating of high spatial frequency presumably activates X-cells while having little effect on the Y-cells. This is exactly the stimulus condition which makes it impossible for observers to judge which eye has received a grating. It follows, then, that successful utrocular discrimination might depend importantly on the activation of Y-cells. This possibility should be testable by determining the effects of flicker rate on utrocular performance; in particular, we would expect an improvement in utrocular discrimination with increasing temporal frequency, for this should favor Y-cells. It is necessary, of course, to select a spatial frequency which falls within the bandwidth of both $\mathrm{X}$ - and $\mathrm{Y}$ cells.

Motivated by these considerations, we measured utrocular discrimination for a 1-cycle/deg and a 6cycle/deg grating which was flickered in counterphase; rate of flicker was varied over a 3-octave range (1.5-12 Hz). The selection of this particular combination of spatial and temporal frequencies was based on the flicker vs. pattern threshold measurements by Kulikowski and Tolhurst (1973) and on the masking studies by Legge (1978). For all test conditions, total exposure duration was $500 \mathrm{msec}$, but within this period, the grating was presented and withdrawn gradually such that the contrast profile over time obeyed a Gaussian function; with the time constant employed, the test grating was at maximum contrast (i.e., $10 \%$ ) for $200 \mathrm{msec}$. In this way, it was possible to minimize the potential contribution of higher temporal harmonics which inherently accompany abrupt stimulus presentation.

The results for two observers are shown in Figure 8, where percent correct is plotted as a function of temporal frequency. The $0-\mathrm{Hz}$ value represents the case where the grating appeared stationary throughout the presentation interval. Note that, for one observer (R.B.), temporal modulation does influence utroc-

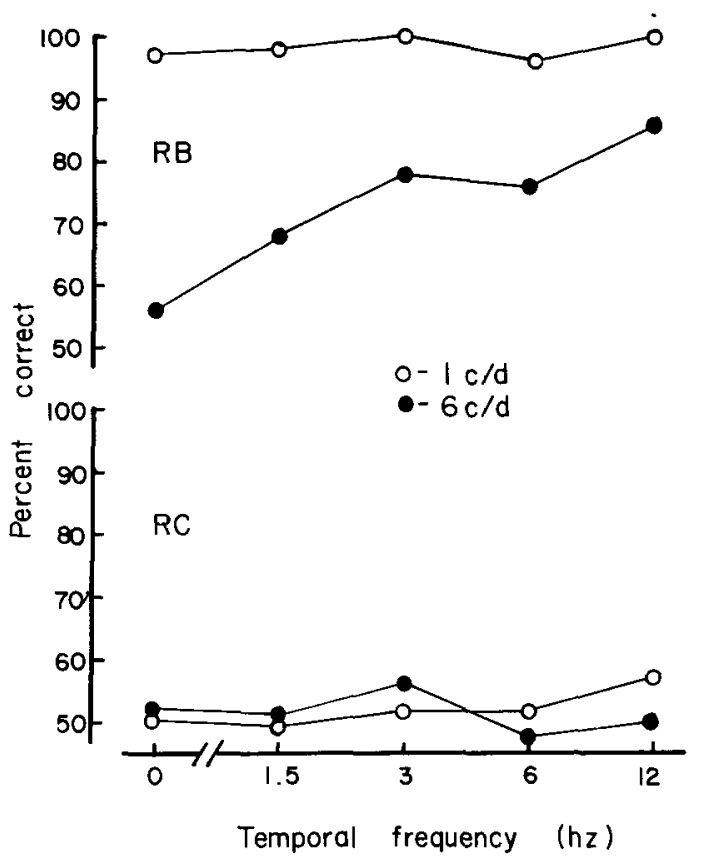

Figure 8. Effects of temporal frequency (counterphase flicker) on utrocular discrimination at two spatial frequencies. 
ular discrimination at 6 cycles/deg, serving to enhance performance by almost $30 \%$ relative to the near-chance performance at $0 \mathrm{~Hz}$. For the 1-cycle/ deg pattern, of course, performance already was near maximum, so we could expect no further improvement with increasing flicker rate. For the other observer (R.C.), however, temporal modulation had no real influence on utrocular performance-the task remained virtually impossible at both spatial frequencies regardless of flicker rate. We have tested two other observers in a similar fashion; one exhibited only a moderate improvement in utrocular performance at 6 cycles/deg with high flicker rates. The other observer showed no consistent effect of temporal frequency. Hence, it appears that temporal frequency, like its counterpart spatial frequency, may play a role in utrocular discrimination for some observers but not for others.

Binocular stimulation. So far, we have focused on stimulus conditions which always involve presentation of a pattern to just one eye or the other. It has been assumed that successful utrocular discrimination must be based on some pattern of neural activity which is uniquely associated with stimulation of one eye as opposed to the other. And conversely, the failure to discriminate eye of origin is assumed to stem from the absence of any such unique pattern of activity, or at least from an inability to utilize that information. Now, it also stands to reason that an observer capable of judging left- from right-eye stimulation should successfully discriminate monocular from binocular stimulation. This would be so because all combinations ${ }^{2}$ of two unique patterns of neural activity (i.e., left-eye and right-eye stimulation) will themselves be different from either component pattern alone. To test this prediction, we performed the following experiment.

On each trial, a stationary vertical grating was presented for $300 \mathrm{msec}$. On half the trials, the pattern went to both eyes simultaneously, while on the remaining trials, the pattern was exposed to either the left eye or the right eye, with equal probabilities. These three types of trials (binocular, monocular left, monocular right) were randomly intermixed, and following each trial the observer judged whether stimulation was binocular or monocular. To avoid potential cues based on contrast differences between monocular and binocular stimulation, grating contrast was randomly varied within a .3-log-unit range $(5 \%$ $10 \%$ ) from trial to trial, completely independently of the exposure condition. The relative phase of the leftand right-eye patterns was carefully adjusted to insure that on binocular trials the fused grating appeared in the plane of depth of the CRT screen. Performance on this task was measured at 1 cycle/deg, a value at which both observers tested excel on utrocular discrimination, and at $8 \mathrm{cycles} / \mathrm{deg}$, where their

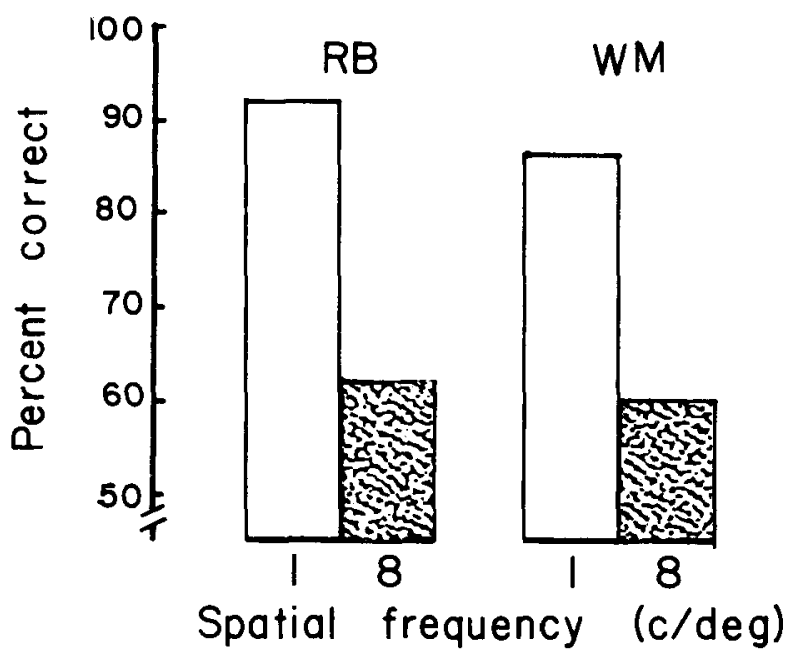

Figure 9. Percent correct for the discrimination of monocular from binocular stimulation at two spatial frequencies.

performance is no better than chance on utrocular discrimination.

The results from this experiment are shown in Figure 9 in the form of histograms. As predicted, at $1 \mathrm{cycle} / \mathrm{deg}$, binocular stimulation could be discriminated from monocular, whereas at $8 \mathrm{cycles} / \mathrm{deg}$, monocular and binocular conditions were often confused.

Next, consider another implication of the assumption we described above, which attributes successful utrocular discrimination to differences in neural activity associated with stimulation of the left eye vs. the right eye. Imagine that stimulation always is binocular but that the two eyes receive patterns which differ along some stimulus dimension. So long as this difference is sufficient to maintain an imbalance in the pattern of neural activity between the two eyes, there would exist some neural basis for judging which eye received a particular visual stimulus, a task that could be characterized as a modified version of utrocular discrimination.

To illustrate this idea, consider the case of contrast disparity between the two eyes. Suppose that on each test trial one eye receives a high-contrast grating of 1 cycle/deg while the other eye receives a very lowcontrast grating of the same spatial frequency; the observer is instructed to report which eye received the higher contrast. If the low-contrast grating were actually below threshold, then the task would be no different from the case of simple utrocular discrimination which, at $1 \mathrm{cycle} / \mathrm{deg}$, would be easy for many observers. Suppose, however, that we systematically increase the contrast of the weaker grating, measuring at each value the observer's ability to judge which eye received the stronger contrast. As this contrast disparity shrinks, the task presumably would become more difficult, owing to the diminish- 
ing cues provided by the difference in neural activity between the two eyes. With this idea in mind, we felt it would be instructive to perform such an experiment as a way of determining just how acute this potential cue might be.

To accomplish this, we arranged for both eyes to receive 1-cycle/deg vertical gratings on every trial. One grating was always of $10 \%$ contrast, and the eye receiving this pattern was varied randomly over trials; the contrast of the other grating always was less than $10 \%$, with the actual value varied over a 1.0-log-unit range in 2-dB steps (.05 log units). At each contrast value, a block of 100 trials was given, with the observer's task being to indicate which eye received the higher contrast.

Figure 10 gives the results for the two observers tested on this task; percent correct is plotted as a function of the difference in contrast between the two eyes. Note that performance deteriorates as this difference becomes increasingly smaller and falls to the level of chance when the two gratings are within 6-8 $\mathrm{dB}$ of one another in contrast. This loss in performance is not due to an inability to discriminate contrast differences of this size, for control trials prove that both observers could judge with perfect accuracy whether a monocularly presented grating was the $10 \%$ pattern or one which was $2 \mathrm{~dB}$ less in contrast. Hence, we must attribute the falloff in performance on this modified utrocular discrimination task to some progressive reduction in the differential neural activity which supports successful discrimination in the case of large contrast disparities.

\section{Part 2}

The results from Part 1 clearly demonstrate that the ability to make eye-of-origin discriminations varies widely among observers. Moreover, those individuals capable of successful discrimination exhibit this knack only for certain stimulus conditions, namely, at lower spatial frequencies. To the extent that the working model outlined in the introduction is reasonable, these results imply that the pattern of neural interactions between the two eyes may differ across the spatial frequency spectrum within some, but not all, individuals. To explore this implication in more detail, we have studied the performance of several observers on other tasks which presumably reflect the involvement of binocular neural interaction. In particular, we measured binocular summation, interocular transfer, and stereoacuity at different spatial frequencies for several observers, including R.B. (who reliably makes utrocular discriminations at low, but not high, spatial frequencies) and R.C. (who fails at utrocular discrimination regardless of spatial frequency). The results from these psychophysical experiments are presented in this part.

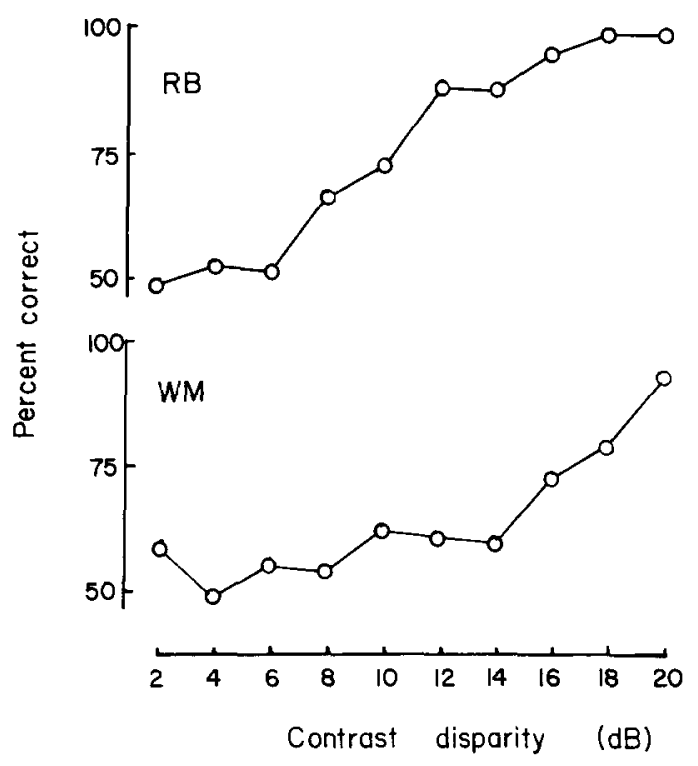

Figure 10. Percent correct for identification of the eye receiving higher contrast. Both eyes received 1-cycle/deg gratings which differed in contrast by the amounts shown along the abscissa. The higher contrast was always $10 \%$.

Binocular summation. On a variety of visual tasks involving threshold measures, binocular performance exceeds monocular performance by an amount greater than that expected on the basis of probability summation (see Blake \& Fox, 1973, for a review of this literature). This superiority of two eyes over one, known as binocular summation, is generally believed to reflect the involvement of neural summation between monocular inputs. The fact that individuals with deficient stereopsis exhibit little, if any, binocular summation (Lema \& Blake, 1977) is consistent with this belief. Previous measurements of binocular summation for contrast thresholds have disclosed no obvious effect of spatial frequency (Blake \& Levinson, 1977; Campbell \& Green, 1965; Lema \& Blake, 1977; Rose, 1978). In view of our present findings, however, we felt it worthwhile to reexamine this possibility, in particular, comparing the amount of binocular summation displayed by R.B. and R.C.

The procedure is described in the Methods section (contrast adjustment method). Adaptation was to an uncontoured display. Thresholds were obtained for the right eye, the left eye, and both eyes. To quantify the amount of binocular summation, a ratio was formed between the contrast threshold measured monocularly (average of the left- and right-eye values) and that measured binocularly; ratio values greater than unity thus reflect lower binocular thresholds. Figure 11 plots this binocular summation ratio for spatial frequencies covering a 3-octave range. The dotted line and open arrow denote a ratio of 1.41 


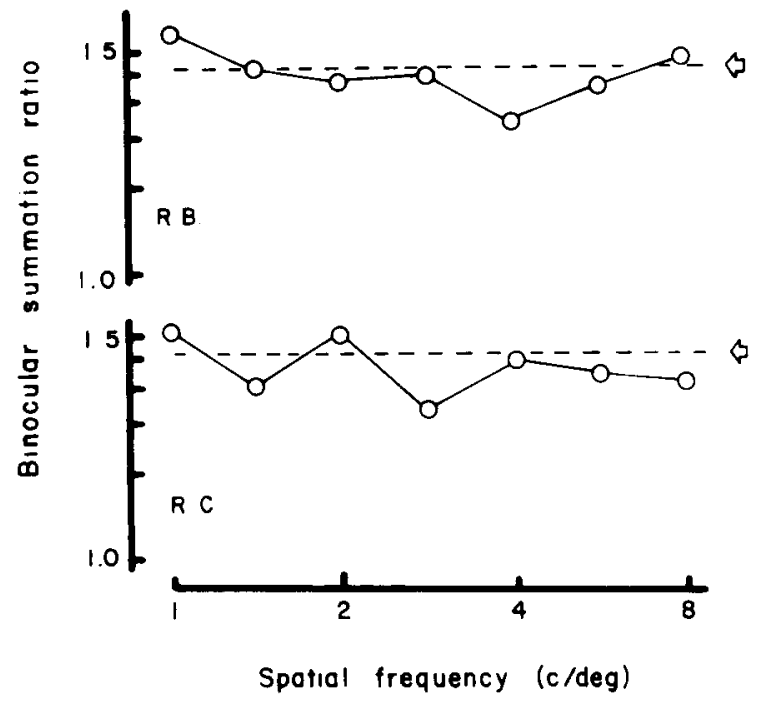

Figure 11. The amount of binocular summation on a contrast threshold task as the function of spatial frequency. Each symbol plots the ratio of the average of the two monocular thresholds (each of which was based on six contrast settings) to the binocular threshold (which was based on six settings). The dotted line and open arrow denote the summation ratio characteristic of performance on this task.

(i.e., $\sqrt{2}$ ), a value which seems to characterize the amount of binocular summation typically found for this sort of task (e.g., Campbell \& Green, 1965). Note that for both observers the binocular summation ratio fluctuates unsystematically about that value, with no tendency for the amount of summation to vary with spatial frequency. Nor is there any notable difference in binocular summation between the two observers, in contrast to their marked differences in performance on the eye-of-origin task (see Figure 1). In summary, there appears to be no simple relationship between performance on the eye-of-origin task and the amount of binocular summation on a contrast threshold task.

Interocular transfer. Next, we turned to another conventional psychophysical gauge of binocularity, the perceptual phenomenon known as interocular transfer. This phenomenon, whereby some aftereffect of visual adaptation of one eye can be observed in the nonadapted eye, implies that the aftereffect occurs at a binocular site within the visual system, where inputs from the two eyes have been combined. There is some evidence that the amount of interocular transfer is related to stereoacuity (Mitchell \& Ware, 1974; Movshon, Chambers, \& Blakemore, 1972), although this relationship has been questioned very recently (Hess, 1978). It does seem clear that stereoblind individuals routinely show a reduced degree of interocular transfer relative to the range found among normal observers (Lema \& Blake, 1977; Wade, 1976; Ware \& Mitchell, 1974). In view of our findings on utrocular discrimination, we wondered whether the amount of interocular transfer exhibited by R.B. and R.C. might depend on the spatial frequency used for adaptation and test. To explore this possibility, we measured the elevation in contrast threshold in one eye produced by prior adaptation of the other eye.

Our procedure was much like that employed in the binocular summation experiment; again, the observer set contrast thresholds using an adjustment method. For this experiment, though, the initial 2-min period involved the continuous presentation of a high-contrast (1.5 log units above threshold) grating to one eye. Following this initial period of adaptation, threshold settings were made using the nonadapted eye. For purposes of comparison, unadapted threshold settings also were obtained by lowering the adaptation contrast to some arbitrary, subthreshold value, such that the observer saw only the uncontoured display during periods of adaptation. Interocular transfer was measured at 1 and at 4 cycles $/ \mathrm{deg}$ following adaptation of the left eye or adaptation of the right eye. We also measured the elevation in contrast threshold produced by adapting and testing the same eye. For all conditions, adaptation and test gratings were of the same spatial frequency.

The results from this experiment are given in Figure 12, which shows the elevation in contrast threshold measured interocularly as a percentage of the aftereffect produced by adaptation and testing of the same eye. All adaptation conditions produced a significant elevation in contrast threshold, but the interocular conditions consistently yielded smaller aftereffects, as evidenced by the failure of the histograms in Figure 12 to reach $100 \%$; this lack of complete interocular transfer seems to be characteristic of all visual aftereffects. Of particular importance for our

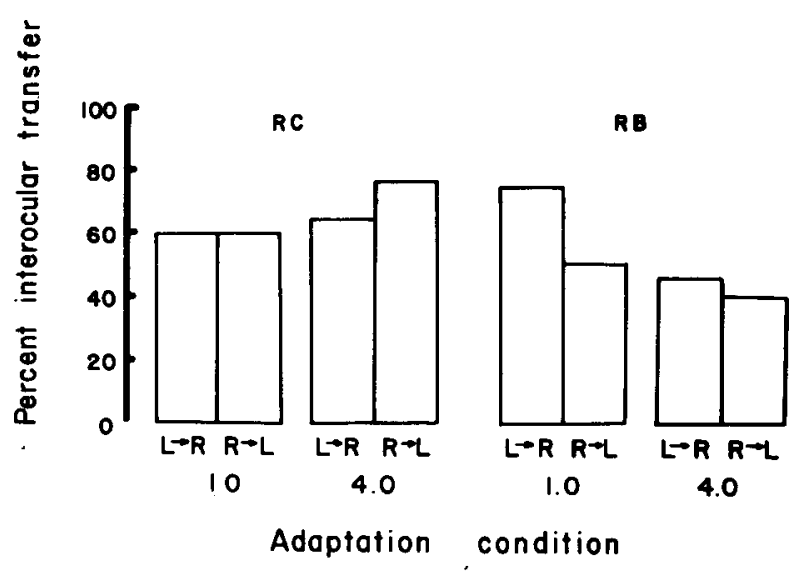

Figure 12. Percent interocular transfer at two different spatial frequencies, 1 and 4 cyles/deg. For the $L \rightarrow R$ conditions, the left eye was adapted to a high-contrast grating and the resulting elevation in contrast threshold $(n=6)$ was measured in the right eye. For the $R \rightarrow L$ condition, adaptation and testing were reversed. Interocular transfer is expressed relative to the threshold $(n=6)$ elevation aftereffect induced and measured in the same eye. 
purposes is the failure to find any effect of spatial frequency. For both observers, the amount of interocular transfer is just about equivalent at the two spatial frequencies, regardless of which eye is tested. Nor is there any striking difference between the amount of transfer for R.B. and R.C. Interocular transfer bears no obvious relationship to discrimination performance on the eye-of-origin task.

Stereoacuity. The final measure of binocular performance studied was stereoacuity, the ability to make judgments of depth based on retinal disparity. As already pointed out, there is a growing conviction in visual science that binocularly innervated cortical neurons play a crucial role in stereopsis. As a corollary to this proposition, it also is assumed that deficiencies in stereopsis reflect a reduction in the density, or proportion, of cortical neurons which can be binocularly excited. Since our utrocular discrimination data suggest that the degree of binocular interaction may depend on spatial frequency, we felt it worthwhile to measure stereoacuity at different spatial frequencies, in order to look for possible correlations between stereoacuity and utrocular performance.

Conventional measures of stereoacuity, such as the three-needle test, do not lend themselves to the study of the effects of spatial frequency on stereopsis. There is, however, a recently described stereoscopic phenomenon which is appropriate for our purposes. This phenomenon, which has been studied by Blakemore (1970) and by Fiorentini and Maffei (1971), is the apparent rotation of a grating in depth about the vertical axis produced by stereoscopically viewing two vertical grating patterns of slightly different spatial frequency. Within limits, the degree of rotation away from the frontoparallel plane is directly related to this spatial frequency difference between the two eyes; the fused grating appears rotated away from the eye receiving the lower spatial frequency. As a measure of stereoacuity, an observer may be given control of the spatial frequency of the grating presented to one eye and instructed to adjust the fused grating until it appears exactly in the frontoparallel plane, with neither the right nor the left side of the pattern in depth. With repeated trials, the average setting provides a measure of constant error, while the variability (standard deviation) of the settings furnishes an index of stereoacuity, with low variability indicating high stereoacuity. In this respect, the logic of the task resembles that of other, more conventional, stereoacuity measures based on equidistance settings (e.g., Ogle, 1950). With our procedure, however, it is straightforward to assess stereoacuity at different spatial frequencies. We obtained frontoparallel settings for gratings at $1,2,4$, and 8 cycles/deg.
Table 1

Stereoacuity (Standard Deviation of Nulling Adjustments, $n=10$ ) for Four Spatial Frequencies and Three Observers

\begin{tabular}{ccccc} 
& \multicolumn{4}{c}{ Spatial Frequency } \\
\cline { 2 - 5 } Observer & 1 & 2 & 4 & 8 \\
\hline R.B. & .023 & .013 & .004 & .048 \\
J.C. & .013 & .009 & .003 & .102 \\
R.C. & .008 & .005 & .007 & .010 \\
\hline
\end{tabular}

Table 1 contains the standard deviations (stereoacuity) for each of three observers at the various spatial frequencies. These values have been normalized for spatial frequency $\left(0 \div\right.$ cycles $\left.\mathrm{deg}^{-1}\right)$, so that the values may be compared directly. First, we should point out that the results at 8 cycles/deg may be of limited use for our purposes; all observers found the task very difficult at this spatial frequency because of the weakness of the stereoscopic sensation of rotation. It was quite frustrating to attempt to bring the fused grating into the frontoparallel plane in the absence of a clear rotation out of this plane. We suspect that this must be responsible, at least in part, for the consistently inferior performance at this high spatial frequency. At the other spatial frequencies, the sensation of depth was quite immediate and compelling, which made the task much simpler. Note, for observers R.B. and J.C., that stereoacuity improves with increasing spatial frequency. This is in contrast to their results on the utrocular discrimination task (see Figure 1), where performance deteriorated with increasing spatial frequency. For observer R.C., whose performance was uniformly poor on the utrocular task, stereoacuity is uniformly good at 1,2 , and 4 cycles/deg. This pattern of results suggests that stereoacuity and utrocular sensitivity may be inversely related. Certainly, our earlier findings (Blake \& Cormack, 1979) on utrocular discrimination in stereoblind individuals are consistent with such a relationship.

\section{DISCUSSION}

In this section, we discuss the methodological implications of our findings, relate those findings to previous studies, and examine some physiological speculations regarding utrocular discrimination.

\section{Methodological Considerations}

The elimination of extraneous cues constitutes a perennial problem in any discrimination task. In utrocular discrimination, this problem is exacerbated by factors associated with halploscopic displays and with potential ocular asymmetries. In our experiments, we are convinced that such factors make no contribution to the pattern of results obtained. For 
one thing, great care was taken in aligning and equating the displays to the two eyes. The procedures followed to accomplish this are described in detail in the Methods section. In addition to those procedural safeguards, the results themselves serve to confirm that potentially confounding variables were controlled adequately. We summarize here some results relevant to the question of extraneous cues.

Despite procedures to equate the right-eye and lefteye displays, it is conceivable that subtle differences (e.g., orientation) could go undetected in a phenomenal match. Then, under forced-choice testing, an observer with excellent visual acuity might be able to use these small differences to make utrocular discriminations. It is not at all obvious, however, why such information would be discernible only at low spatial frequencies. While it is known that certain spatial frequencies are more detectable than others (e.g., Schade, 1956), the effect of spatial frequency on utrocular discrimination bears absolutely no relationship to this contrast-sensitivity function. Even more to the point, performance is unaffected when stimulation is confined to extrafoveal portions of the retina where spatial acuity is lower. These considerations rule out the involvement of slight stimulus differences between the two displays which, in order to be detected, would require good visual acuity. These considerations apply equally to arguments based on acuity differences between the two eyes which, in the case of sine-wave gratings, manifest themselves as contrast differences between the retinal images. In any event, in our previous work (Blake \& Cormack, 1979), we found that successful utrocular discrimination survived deliberate trial-to-trial randomization of stimulus contrast.

Our results also tend to rule out as a cue slight differences in visual direction which can arise from fixation disparity. For one thing, the rapid apparent motion accompanying counterphase flicker would obliterate the position information conveyed by fixation disparity, yet for at least some observers, performance improves with flicker. For another thing, utrocular discrimination with horizontal gratings is equivalent to that with vertical, despite the fact that vertical fixation disparities (which could provide cues only for horizontal contours) are extremely small relative to horizontal fixation disparities (Ogle, 1950). Finally, there is no reason to expect fixation disparity to be most effective at low spatial frequencies.

A skeptic might wonder whether observers were employing deliberate viewing strategies which would render the task trivially simple. Such strategems might include closing one eye, viewing with crossed eyes, or altering an image by squinting one eye. The more gracious critic, noting that the authors were the two principal observers, might point out that chronic suppression or lateral phorias could mimic these behaviors. These possibilities seem remote for several reasons. In terms of our procedures, the cover/uncover test was used specifically to compensate for any phorias, and observers were instructed to fixate the center of the display, to keep both eyes open, and to initiate a trial only when these conditions were met. The observers reported no difficulty in maintaining fusion of the two CRT displays and noted no phenomenal changes in the appearance of the pattern from trial to trial. A pattern was seen on every trial, which would not be the case if one eye were chronically suppressed. Also, recall that observers could discriminate monocular from binocular stimulation and could judge which eye received higher contrast when both eyes received a pattern. Neither of these would be possible if either eye were suppressed.

Finally, from the above discussion, it should be obvious that any extraneous cue or viewing strategy mediating utrocular discrimination must be sensitive to spatial frequency. We have been unable to find or even conceive of a cue which would survive our experimental manipulations and which would be effective at low, but not at high, spatial frequencies.

\section{Relation to Previous Work}

The present series of experiments explored several variables also studied in earlier work. Our results showing no effect of cycloplegia agree with those of Enoch et al. (1969) and Smith (1945). Similarly, the absence of an effect of duration is in agreement with the findings of Enoch et al. and Pickersgill (1961). With respect to the effects of practice on utrocular discrimination, Enoch et al. and Thelin and Altman (1929) reported moderate improvement from training with feedback, especially among inexperienced observers. In the present study, no systematic effects of practice are revealed. This could be due to the fact that our observers were given many $(>200)$ unrecorded practice trials before any data were collected. It might be noted that a few observers reported a growing awareness of the unique sensation upon which they ultimately based their discrimination.

Our results reveal no difference between central and peripheral stimulation, a finding which is at variance with the results of Enoch et al. (1969) and Smith (1945). Enoch et al. found that utrocular discrimination is superior in the central retina, but primarily in the case of short exposure durations. They concluded that with longer durations, eye movements could bring the target to the central retina. There is, however, a potential confound in their study. Their data for the central retina were obtained under conditions of location certainty, whereby, on each trial, observers knew exactly where the stimulus would appear. For their peripheral stimulus conditions, they employed multiple target locations, thereby in- 
troducing location uncertainty. It is conceivable that location uncertainty, not retinal eccentricity, produced the observed effect.

Smith (1945) also reported that central stimulation yields better performance than peripheral stimulation. While his data are not confounded with location uncertainty, Smith himself speculated that fixation disparity might have been a source of error in the results, reasoning that small location differences between the two eyes would be more noticeable in the fovea than in the periphery. In view of the nature of Smith's stimuli (spots of light), this is a likely possibility.

In agreement with all previous work on utrocular discrimination, we find evidence for large individual differences. At low spatial frequencies, observers range along a continuum from nearly perfect discrimination to chance performance. At high spatial frequencies, no individual differences are found; no observer could make the discrimination at these values. In relation to earlier work, we do not know precisely the spatial frequency content of the targets used in those studies. We have shown, though, that the presence of high spatial frequencies does not interfere with utrocular discrimination so long as low spatial frequency components are present. It is certainly possible that the successful observers in previous work were responding to low spatial frequency components of the targets.

\section{Theoretical Implications}

The data reported here have forced us to reexamine some of our conceptions regarding utrocular discrimination. These conceptions initially seemed quite reasonable and, indeed, stimulated our original interest in the problem. It stands to reason that utrocular discrimination must be mediated by a mechanism which shows differential responses to inputs from the two eyes. Clearly, it cannot be based on cells whose responses to inputs from the two eyes are identical. Initially, we speculated that this mechanism might consist of monocularly innervated neurons. This speulation was reinforced by the finding that stereoblind individuals, who show no binocular summation and very little interocular transfer, have no difficulty making utrocular judgments (Blake \& Cormack, 1979). The absence of binocular summation and interocular transfer, as well as stereoblindness itself, are generally interpreted as reflecting a low proportion of binocular cells and a concomitantly high proportion of monocular cells. From these considerations, we inferred that among observers with normal stereopsis the same conditions which optimize utrocular discrimination should yield reduced binocular summation, interocular transfer, and stereoacuity. Our results, however, show absolutely no tradeoff between utrocular performance, on the one hand, and binocular summation and interocular transfer, on the other. This is particularly intriguing in the case of interocular transfer, for it is widely believed that the failure of aftereffects to transfer completely reflects the involvement of monocular neurons. While we found incomplete interocular transfer of the threshold elevation aftereffect, the degree of transfer was equivalent at low and high spatial frequencies even for an observer (R.B.) who shows excellent utrocular discrimination at low but not at high spatial frequencies. Nor did the degree of transfer for this observer differ from that of an observer (R.C.) who fails at utrocular discrimination at all spatial frequencies. From this we must conclude that the mechanism underlying utrocular discrimination differs from that responsible for the incompleteness of interocular transfer.

With respect to our measures of stereoacuity, there is some suggestion that stereopsis varies with spatial frequency for those observers whose utrocular discrimination depends on spatial frequency. For observer R.C., who shows no utrocular discrimination, there appears to be no relationship between stereoacuity and spatial frequency. We are reluctant, however, to emphasize this finding, for two reasons. In the first place, the relationship is extremely modest and, in the second, all three observers exhibit excellent stereopsis on other more conventional tests. Still, it would seem worthwhile to examine in greater detail the influence of spatial frequency on stereopsis, particularly in a larger sample of observers. The procedures we have developed are uniquely suited for this purpose.

Another tempting speculation centers on the possible differential contributions of $\mathrm{X}$ - and $\mathrm{Y}$-cells to utrocular discrimination, an idea which motivated us to examine the effects of temporal frequency. In spite of the modest, but definite, effects obtained, we have encountered difficulties in developing an interpretation of utrocular performance based on these two classes of cells. The receptive field properties of $Y$ cells led us to believe that they, rather than X-cells, might mediate utrocular discrimination. But, if this were true, it would imply that observers completely unsuccessful at utrocular discrimination (e.g., R.C.) either lack the normal complement of Y-cells or possess $\mathrm{Y}$-cells with unusual properties which fail to supply the requisite information. Now, we are aware of the fact that $Y$-cells can be profoundly influenced by early visual experience (e.g., Sherman, Hoffman, \& Stone, 1972). Yet it would seem that such a dramatic anomaly in Y-cells should be expressed in other visual functions, besides utrocular discrimination. But this is not the case, as evidenced by extensive visual testing and performance on many other psychophysical tasks. The notion that Y-cells mediate utrocular discrimination also implies that $\mathrm{X}$-cells and 
Y-cells differ with respect to their ocular dominance profiles; specifically, Y-cells would have to exhibit a greater degree of monocularity. A careful review of the relevant neurophysiological literature fails to reveal such a difference. For these reasons, it seems premature to emphasize the possible differential involvement of $\mathrm{X}$ - and $\mathrm{Y}$-cells in utrocular discrimination.

\section{Conclusion}

Our experiments demonstrate that the ability to judge which eye has received stimulation is uninfluenced by a variety of factors. In the case of our studies, these include duration, contrast, orientation, retinal locus, and practice, none of which alters performance on the task. On the other hand, we have for the first time identified a variable (i.e., spatial frequency) which does exert strong control over utrocular discrimination. A high percentage of observers can perform this task at low spatial frequencies, while none can do so at high spatial frequencies. We have also discovered that temporal frequency may exert at least weak control over utrocular discrimination for some observers. Identifying such controlling variables represents an important step, for it now allows us to manipulate utrocular discrimination and thus observe its relationship to other phenomena. This also makes it possible to assess the involvement of extraneous cues, as our earlier discussion has shown, and to test specific hypotheses regarding the basis of utrocular discrimination.

\section{REFERENCES}

Barlow, H. B., Blakemore, C., \& Pettigrew, J. D. The neural mechanism of binocular depth discrimination. Journal of Physiology, 1967, 193, 327-342.

BarRett, G. V., \& Williamson, T. R. Judging with which eye one is viewing a three dimensional scene. Perceptual \& Motor Skills, 1965, 21, 455-458.

Berkley, M. A., Kitterle, F., \& Watkins, D. W. Grating visibility as a function of orientation and retinal eccentricity. Vision Research, 1975, 15, 239-244.

Blake, R., \& Cormack, R. Psychophysical evidence for a monocular visual cortex in stereoblind humans. Science, 1979, 203, 274-275.

Blake, R., \& Fox, R. The psychophysical inquiry into binocular summation. Perception \& Psychophysics, 1973, 14, 161-185.

BlaKe, R., \& HiRsCH, H. V. B. Binocular depth discrimination in normal and specially-reared cats. Science, 1975, 190, 1114-1116.

Blake, R., \& Levinson, E. Spatial properties of binocular neurones in the human visual system. Experimental Brain Research, 1977, 27, 221-232.

Blakemore, C. A new kind of stereoscopic vision. Vision Research, 1970, 10, 1181-1199.

Brindley, G. S. Physiology of the retina and visual pathway (2nd ed.). Baltimore: Williams \& Wilkins, 1970.

Campbell, F. W., \& Green, D. G. Monocular versus binocular visual acuity. Nature, 1965, 208, 191-192.

Campbell, F. W., Howell, E. R., \& Robson, J. G. The appear- ance of gratings with and without the fundamental Fourier component. Journal of Physiology (London), 1971, 217, 17-18P.

Enoch, J., Goldmann, H., \& Sunga, R. The ability to distinguish which eye was stimulated by light. Investigative Ophthalmology, 1969, 8, 317-331.

Fiorentini, A., \& MAFFeI, L. Binocular depth perception without geometrical cues. Vision Research, 1971, 11, 1299-1305.

GREGORY, R. L. Eye and brain, the psychology of seeing (3rd ed.). New York: McGraw-Hill, 1978.

Harmon, L. D., \& Julesz, B. Masking in visual recognition: Effects of two-dimensional filtered noise. Science, 1973, 180, 1194-1197.

Hess, $R$. Interocular transfer in individuals with strabismic amblyopia; a cautionary note. Perception, 1978, 7, 201-205.

IKeDA, H., \& Wright, M. J. Processing of spatial and temporal information in the visual system. In F. O. Schmitt \& F. G. Worden (Eds.), The neurosciences, third study program. Cambridge, Mass; MIT Press, 1974.

KELLY, D. H. Effects of sharp edges on the visibility of sinusoidal gratings. Journal of the Optical Society of America, $1970,60,98-103$

Kulıkowskı, J. J., \& Tolhurst, D. J. Psychophysical evidence for sustained and transient detectors in human vision. Journal of Physiology, 1973, 232, 149-162.

LEGGE, G. E. Sustained and transient mechanisms in human vision: Temporal and spatial properties. Vision Research, 1978, $18,69-81$

LEMA, S. A., \& Blake, R. Binocular summation in normal and stereoblind humans. Vision Research, 1977, 17, 691-695.

Mitchell, D. E., \& WARE, C. Interocular transfer of a visual aftereffect in normal and stereoblind humans. Journal of Physiology, 1974, 236, 707-721.

Movshon, J. A., Chambers, B. E. I., \& Blakemore, C. Interocular transfer in normal humans and those who lack stereopsis. Perception, 1972, 1, 483-490.

Nikara, T., Bishop, P. O., \& Pettighew, J. D. Analysis of retinal units in cat striate cortex. Experimental Brain Research, 1968, 6, 353-372.

OGLE, K. N. Researches in binocular vision. New York: Hafner, 1950.

Packwood, J., \& Gordon, B. Stereopsis in normal domestic cat, Siamese cat, and cat raised with alternate monocular occlusion. Journal of Neurophysiology, 1975, 38, 1485-1499.

Pickersgill, M. J. On knowing with which eye one is seeing. Quarterly Journal of Experimental Psychology, 1961, 13, 168-172.

Rose, D. Monocular versus binocular contrast thresholds for movement and pattern. Perception, 1978, 7, 195-200.

Schade, O. H. Optical and photoelectric analog of the eye. Journal of the Optical Society of America, 1956, 46, 721-739.

Sherman, S. M., Hofrman, K. P., \& Stone, J. Loss of a specific cell type from dorsal lateral geniculate nucleus in visually deprived cats. Journal of Physiology, 1972, 35, 532-541.

SмITH, S. Utrocular or 'which eye' discrimination. Journal of Experimental Psychology, 1945, 35, 1-14.

Stromeyer, C. F., \& Julesz, B. Spatial-frequency masking in vision: Critical bands and spread of masking. Journal of the Optical Society of America, 1972, 62, 1221-1232.

Templeton, W. B., \& Green, F. A. Chance results in utrocular discrimination. Quarterly Journal of Experimental Psychology, 1968, 20, 200-203.

Thelin, E., \& Altman, E. R. Identification of monocular functions. Journal of Experimental Psychology, 1929, 1, 79-87.

WADE, N. J. On interocular transfer of the movement after-effect in individuals with and without normal binocular vision. Perception, 1976, 4, 85-95.

WARE, C., \& Mitchell, D. E. On interocular transfer of various visual aftereffects in normal and stereoblind observers. Vision Research, 1974, 14, 731-734. 


\section{NOTES}

1. The term "utrocular" stems from the Latin root uter, meaning which one of two. It appears that Smith (1945) was the first to employ this term in reference to the problem of distinguishing which eye was stimulated. In this paper, we shall use the terms "utrocular" and "eye of origin" interchangeably.

2. An interesting twist on the utrocular discrimination paradigm was employed by Templeton and Green (1968). In that experiment, subjects viewed two circular light targets stereoscopically, such that each eye saw just one of the targets. Care was taken to properly situate the two half-images such that fixation disparity was minimal; the resulting percept was a single spot of light. From trial to trial, one of the two half-images was briefly extinguished and the subjects were asked to guess which eye's view was removed. Even with training and feedback, the subjects were unable to perform above the chance level.
3. One of the reviewers points out that the input to the pupillomotor centers probably originates largely from the retinal W-cells. Because cells in this category tend to have poorer spatial resolving properties and hence to respond selectively to lower spatial frequencies, the reviewer suggests that the W-cell pathway may provide a plausible neural channel for utrocular discrimination. This is an intriguing hypothesis.

4. The only exception to this rule would be some situation where stimulation of one, but only one, of the eyes evoked no neural activity, in which case utrocular discrimination would boil down to a judgment based simply on the presence or absence of activity on each trial. In this instance (which could arise if the two eyes were to differ greatly in terms of sensitivity), monocular and binocular stimulation would be indiscriminable.

(Received for publication December 18, 1978; revision accepted March 15, 1979.) 